\title{
Brightness enhancement and the Talbot level in stationary gratings
}

\author{
JAMES T. WALKER \\ University of Missouri-St. Louis, St. Louis, Missouri 63121
}

\begin{abstract}
At low spatial frequencies, the perceived brightness of the light phase of a stationary square-wave grating is greater than the brightness of a solid field of equal physical luminance. That increase in the perceived brightness of a grating at low spatial frequencies is analogous to the brightness enhancement observed in a flickering light at low temporal frequencies. At or above the critical spatial frequency-the visual resolution threshold-the brightness of a grating is determined by its space-average luminance, just as the brightness of a flickering light at or above the critical flicker frequency is determined by its time-average luminance in accordance with Talbot's law. Thus, Talbot's law applies in the spatial as well as the temporal domain. The present study adds to the evidence that temporal and spatial frequency play analogous roles in some aspects of brightness vision.
\end{abstract}

Brightness enhancement, the increased brightness of a flickering light in comparison with a steady light, has been demonstrated in a great many situations involving luminance changes in time (e.g., Bartley, 1938, 1951; Levinson, 1968). At some temporal frequencies, the perceived brightness of a flickering light is considerably greater than the brightness of a steady light of equal physical luminance. Maximum brightness enhancement occurs at a temporal frequency of about $10 \mathrm{~Hz}$. At some higher frequency, the brightness of a flickering light decreases to that of a steady light of equal objective luminance; at still higher frequencies, the brightness decreases below that of a steady light. At the critical flicker frequency-about $40 \mathrm{~Hz}$, depending on luminance, light-dark ratio, and some other parameters-the temporal resolution of the visual system fails and the flickering light appears steady. Talbot's law holds that the brightness of a flickering light at or above the critical flicker frequency is determined by its time-average luminance, and that level of luminance has been called the Talbot level.

In the detection of flicker, a threshold enhancement effect also occurs at a temporal frequency of about $10 \mathrm{~Hz}$. De Lange (1958) measured the flicker detection threshold in terms of the luminance contrast' between the light and dark phases of a flickering light which the observer could just discriminate from a steady appearing light. For high levels of time-average luminance, flicker detection thresholds were lowest - that is, maximally enhanced-at

A portion of this paper was presented at the annual meeting of the American Psychological Association, New Orleans, Louisiana, August 1974. I thank Virginia Gossage for assistance in data collection. about $10 \mathrm{~Hz}$. By analogy with the earlier demonstrations of brightness enhancement, de Lange's results might have been expected, although the detection of flicker and the perceived brightness of a flickering light are not identical phenomena.

The concept of frequency is useful in the spatial as well as the temporal domain. For example, a grating pattern consisting of alternating light and dark bars can be described in terms of its spatial frequency-that is, the number of light-dark cycles per degree of visual angle. Campbell and Robson (1968) measured the detectability of stationary sinewave gratings as a function of spatial frequency. Detection thresholds of gratings were measured in terms of luminance contrast ${ }^{1}$ between the light and dark phases of a grating which the observer could just discriminate from a solid appearing field. Under some conditions, thresholds were lowest at spatial frequencies of about $3 \mathrm{c} / \mathrm{deg}$ of visual angle, increasing substantially at lower frequencies and enormously at higher frequencies. Thus, a kind of threshold enhancement occurs as a function of spatial frequency. Although Campbell and Robson do not refer to the earlier work of de Lange, their results in the spatial domain appear quite analogous to his earlier results in the temporal domain.

In the perception of flickering light, brightness enhancement and flicker detection threshold enhancement occur at some temporal frequencies. In the perception of stationary gratings, enhancement of the grating detection threshold occurs as a function of spatial frequency, but no analogous brightness enhancement effect has been reported in gratings. Thus, the question arises whether brightness enhancement occurs in stationary gratings as a function of spatial frequency. A further question arises regarding 
Talbol's law: Since the visual resolution threshold of a grating constitutes a critical spatial frequency analogous to the critical flicker frequency of a flickering light, does Talbot's law apply in the spatial as well as the temporal domain? That is, does the spaceaverage luminance of a grating determine its brightness at or above the critical spatial frequency? The present study was designed to measure the brightness of square-wave gratings across a range of spatial frequencies in order to answer those questions.

\section{EXPERIMENT 1}

In this experiment, the observers adjusted the luminance of an annulus subtending $2^{\circ}$ of visual angle to match the constant luminance of a vertical grating pattern subtending $1^{\circ}$. The observer's view of the annulus and grating is shown in Figure 1A.

\section{Method}

Observers. The writer and a colleague, both with corrected vision, served as observers.

Apparatus. The square-wave gratings were photographed on Kodalith film. The gratings were presented in a circular field $7 / 8 \mathrm{in} .(2.22 \mathrm{~cm})$ in diameter at a viewing distance of $50 \mathrm{in}$. $(127 \mathrm{~cm})$ so as to subtend a visual angle of $1^{\circ}$. All gratings were oriented vertically. The spatial frequency of the gratings, defined here as the number of white-black cycles per degree of visual angle, ranged from .5 to $128 \mathrm{c} / \mathrm{deg} .{ }^{2}$ For a spatial frequency of $.5 \mathrm{c} / \mathrm{deg}$, the white phase of a white-black cycle subtends $1^{\circ}$ of visual angle, so for that spatial frequency the grating field was solid white. The gratings were transilluminated by a white surface lighted by a projector powered by a constant-voltage transformer. The luminance of the white phase of the gratings was $50 \mathrm{~mL}$, as measured by a Macbeth illuminometer. The gratings were somewhat more than $50 \%$ black, the space-average luminance of the highest-frequency grating being $22.3 \mathrm{~mL}$. A white annulus subtending $2^{\circ}$ of visual angle surrounded the grating field. The annulus and grating field were presented simultaneously at equal viewing distances in the two channels of a Iwo-way mirror device and were viewed binocularly. The annulus was lighted by a separate projector, powered as above, and the observer could adjust its luminance by operating a switch controlling a motor-driven iris diaphragm. The luminance of the annulus was monitored by a resistance photocell and recorded automatically. The system was calibrated using a Macbeth illuminometer.

Procedure. Nine different gratings were used having spatial frequencies progressing by powers of 2 from .5 to $128 \mathrm{c} / \mathrm{deg}$ (see Figure 1A). The observer's task was to adjust the luminance of the annulus so that its brightness matched that of the bright phase of the grating. At those spatial frequencies above the resolution threshold-where no lines could be seen--the observer adjusted the annulus to match the overall brightness of the grating field. Each observer made four practice adjustments, in ADDA order, using the $8 \mathrm{c} / \mathrm{deg}$ and the $.5 \mathrm{c} / \mathrm{deg}$ gratings. The gratings were then presented in a different random order to each observer, who made four brightness matches in ADDA order for each grating.

\section{Results}

The results are shown graphically in Figure $1 \mathrm{~A}$. Since means and variances of lumnance measures tend to be correlated, the data were transformed

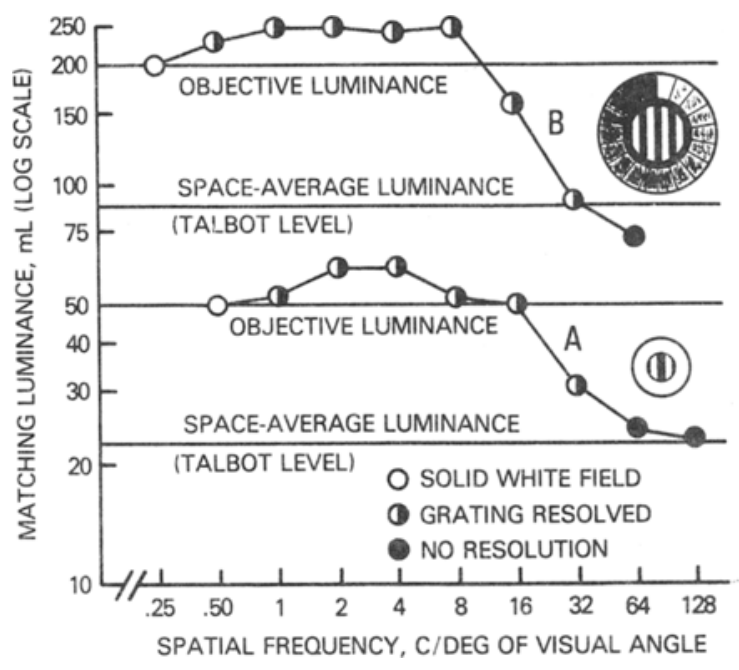

Figure 1. Brightness-frequency functions in Experiment $1(\mathrm{~A})$ and Experimeni $2(B)$. Brightness enhancement oceurs at low spatial frequencies.

logarithmically and a repeated-measures analysis of variance carried out. The effect of spatial frequency was highly significant, $F(8,8)=25.95, p<.001$. Comparing the mean of the brightness matches at spatial frequencies of 2 and $4 \mathrm{c} / \mathrm{deg}$ with the brightness of the solid field at $.5 \mathrm{c} / \mathrm{deg}$ shows that significant brightness enhancement occurred, $F(1,8)=$ $5.89, p<.05$. At the highest spatial frequency, $128 \mathrm{c} / \mathrm{deg}$, the mean brightness match of $23.05 \mathrm{~mL}$ was very close to the space-average luminance of that grating, $22.3 \mathrm{~mL}$, $\mathrm{t}(1)=.52$. Thus, brightness enhancement occurred at low spatial frequencies, while at or above the critical spatial frequency-the visual resolution threshold-the perceived brightness of a grating was determined by its space-average luminance.

\section{EXPERIMENT 2}

This experiment was designed to measure the relationship between grating brightness and spatial frequency using a higher level of luminance, different apparatus and methods, and different observers.

\section{Method}

Observers. Fifteen men and four women, students in an undergraduate perception course, participated as a course requirement. All observers had previously taken part in experiments requiring brightness matching-including an experiment on brightness enhancement of flickering light-but no observer had had any previous experience with brightness measurements of gratings.

Apparatus. The gratings, described in Experiment 1, were projected on a white disk 6 in. $(15.24 \mathrm{~cm})$ in diameter at a viewing distance of $14.3 \mathrm{ft}(4.36 \mathrm{~m})$. Thus, the gratings appeared in a circular field subtending $2^{\circ}$ of visual angle. The spatial frequency of the gratings progsessed by powers of 2 from .25 (solid field) to $64 \mathrm{c} / \mathrm{deg}$ (see Figure $1 \mathrm{~B}$ ). The luminance of the white phase 
of the gratings was $200 \mathrm{~mL}$, as measured by a Macbeth illuminometer. A black annulus of flocked paper subtending $2.6^{\circ}$ of visual angle surrounded the grating field. The black annulus was, in turn, surrounded by a $4.3^{\circ}$ annulus consisting of a graded series of 18 comparison stimuli, Coloraid gray papers, ranging from white to black (see Figure 1B). The comparison stimuli were continuously lighted by a projector, and their luminances ranged from 9 to $450 \mathrm{~mL}$. The black annulus, described above, was intended to reduce the effects of brightness contrast between the comparison stimuli and the grating field. The comparison stimuli were presented against a background with a luminance of $68 \mathrm{~mL}$. Each of the comparison steps was identified by number, 1 through 18, printed on the background so that the observer could indicate the comparison brightness most closely matching the brightness of the grating.

Procedures. The observer's task was to indicate, for each of the gratings, which of the comparison stimuli most closely matched the brightness of the white phase of the grating. Where the grating could not be resolved, the observer matched the overall brightness of the grating. Each grating was presented twice to each observer in a random order counterbalanced within observers.

\section{Results}

The results are shown in Figure 1B. The data were transformed logarithmically and a repeated-measures analysis of variance carried out. The effect of spatial frequency was highly significant, $F(8,44)=109.05$, $\mathrm{p}<.001$. Comparing the mean of the brightness matches at frequencies of 1 through $8 \mathrm{c} / \mathrm{deg}$ with the mean at $.25 \mathrm{c} / \mathrm{deg}$ shows that brightness enhancement occurred, $F(1,144)=15.64, p<.001$. At the highest spatial frequency, $64 \mathrm{c} / \mathrm{deg}$, the mean brightness match of $74.13 \mathrm{~mL}$ was significantly less than the space-average luminance of that grating, $89.2 \mathrm{~mL}$, $\mathrm{t}(18)=2.58, \mathrm{p}<.02$.

\section{GENERAL DISCUSSION}

Brightness enhancement at low spatial frequencies occurred in both experiments in the present study. Although different luminance levels, apparatus, and procedures were used in those experiments, the maximum amount of brightness enhancement observed in each was exactly $.09 \log$ unit (23.03\%).

In Experiment 1, the mean luminance match for the highest spatial frequency did not differ significantly from the space-average luminance of that grating. Thus, it appears that Talbot's law applies in the spatial as well as the temporal domain. ${ }^{3}$ However, the results of Experiment 2 do not so clearly support the application of Talbot's law, since the mean brightness match for the highest-frequency grating was significantly below its space-average luminance in that experiment. In Experiment 2, each grating was surrounded by a series of comparison stimuli ranging in luminance from 9 to $450 \mathrm{~mL}$. It seems likely that the brightness of the highest frequency-which was above the resolution threshold, and thus appeared a uniform gray-was darkened by contrast with the very bright surrounding comaprison luminances. It might also be argued that the very dark comparison luminances might have lightened the brightness of the highest-frequency grating. But Heinemann (1955) has shown that lower inducing luminances lighten a test field less than higher inducing luminances darken that same test field. Thus, darkening - rather than lightening - of the highest-frequency grating is to be expected through the process of brightness contrast in Experiment 2. The data indicate that no comparable brightness contrast occurred in Experiment 1. In that experiment, the observer adjusted the luminance of the annulus so that its brightness matched that of the gratings. For gratings of high spatial frequencies, the matching luminance of the annulus was seldom much greater than the space-average luminance of the gratings; as a result, less brightness contrast probably occurred than in Experiment 2.

If the highest-frequency grating in Experiment 2 was darkened by brightness contrast with the surrounding comparison stimuli, it seems reasonable to ask whether any of the low-frequency gratings were similarly darkened. Clearly, no such darkening occurred in the low-frequency gratings (see Figure 1B); on the contrary, significant brightness enhancement occurred across a range of low spatial frequencies. Even for the solid field, where no brightness enhancement occurred, there is no suggestion of any darkening. The following possible explanation can be offered. The brightest comparison stimulus had a luminance of $450 \mathrm{~mL}$, the solid field a luminance of $200 \mathrm{~mL}$, and the space-average luminance of the highest-frequency grating was $89.2 \mathrm{~mL}$. Since the brightest comparison stimulus was 5.04 times as bright as the space-average luminance of the highestfrequency grating and only 2.25 times as bright as the solid field, that grating should have been subject to greater darkening than the solid field. The black annulus between the comparison stimuli and the grating field in Experiment 2 seems to have eliminated any darkening of the solid field through brightness contrast, but the presence of that annulus was not sufficient to eliminate the darkening of the highestfrequency grating.

While identical amounts of brightness enhancement occurred in Experiments 1 and 2, the results of the second experiment are less in accordance with Talbot's law than are the results of the first. The darkening of the highest-frequency grating in Experiment 2 through brightness contrast with the surrounding comparison stimuli provides a possible explanation of the observed deviation from Talbot's law. The apparatus and procedures used in Experiment 1 probably represent a superior methodology, since brightness contrast between the grating field and the matching luminances seems to have been minimal in that experiment.

The preceding disucssions of brightness contrast have been concerned with the contrast between the grating field and the surrounding comparison stimuli. 
Brightness contrast within gratings- that is, contrast between the light and dark phases of the grating patterns-probably plays an important role in the present phenomena. Indeed, the present brightness enhancement effect can be considered a species of simultaneous-and perhaps successive-brightness contrast phenomenon. The white lines of a grating are lightened by simultaneous contrast with the black lines, and thus the light phase of a grating appears brighter than a solid field of equal physical luminance. It seems likely that successive contrast also enters into determining the brightness of gratings in that eye movements may bring about temporal interactions between the brightness of images and afterimages.

Brightness enhancement occurs at lower spatial frequencies, but at higher frequencies the light phase of a grating appears less bright than a solid field of equal luminance. Thus, contrast gives way to assimilation at higher spatial frequencies. Helson (1963) reports that wide black or white lines respectively lighten or darken a medium gray background through brightness contrast, while narrow black or white lines respectively darken or lighten a gray background through assimilation. The stimuli and methods were very different in the present study, but the results are largely consistent with Helson's earlier findings. In a grating pattern, the ultimate assimilation occurs at the critical spatial frequencythe resolution threshold-where the brightness of a grating is determined by its space-average luminance.

Brightness enhancement occurs in stationary square-wave gratings as a function of spatial frequency and in flickering lights as a function of temporal frequency. Talbot's law has been shown to apply in the spatial as well as the temporal domain. The brightness-frequency functions, the relationships between perceived brightness and temporal or spatial frequency, are very similar for flickering lights and stationary gratings. Thus, the present study provides further evidence of the analogous roles that time and space play in some aspects of brightness vision.

\section{REFERENCES}

Bartley, S. H. Subjective brightness in relation to flash rate and the light-dark ratio. Journal of Experimental Psychology, 1938, 23. 313-319.

BarTley, S. H. Brightness enhancement in relation to target intensity. Journal of Psychology, 1951, 32, 57-62.

Campbell, F. W., \& Robson, J. G. Application of Fourier analysis to the visibility of gratings. Journal of Physiology (London), 1968, 197, 551-566.

DE LANGE, H. Research into the dynamic nature of the human fovea-cortex systems with intermittent and modulated light. 1. Attenuation characteristics with white and colored light. Journal of the Optical Society of America, 1958, 48, 777.784.

Heinemann, E. G. Simultaneous brightness induction as a function of inducing- and test-field luminances. Journal of Experimental Psychology, 1955, 50, 89-96.

Helson, H. Studies of anomalous contrast and assimilation. Journal of the Optical Society of America, 1963, 53, 179-184.

Levinson, J. Z. Flicker fusion phenomena. Science, 1968, 160. $21-28$.

\section{NOTES}

1. The contrast ratio, $\mathrm{C}$, is defined by the following formula:

$$
\mathrm{C}=\left(\mathrm{L}_{\max }-\mathrm{L}_{\text {min }}\right) /\left(\mathrm{L}_{\max }+\mathrm{L}_{\text {min }}\right) \text {, }
$$

where $L_{\max }$ and $L_{\min }$ are, respectively, the maximum and minimum luminances in a visual display, that is, the luminances of the light and dark phases of a grating or the light and dark phases of a flickering light.

2. Through Fourier analysis, a square-wave grating can be analyzed into a number of sine-wave components of progressively higher spatial frequencies (Campbell \& Robson, 1968). Thus, from the standpoint of Fourier analysis, a square-wave grating contains many sine-wave components, and thus contains many spatial frequencies. In the present context, however, the number of whiteblack cycles per degree of visual angle will be taken as the spatial frequency of the square-wave gratings.

3. Using a laser interference pattern, which produces a very high level of modulation on the retina, Tom Cornsweet has found that Talbot's law breaks down with very fine gratings above the resolution threshold. Such a fine grating, although above the resolution threshold, appears darker than an effectively homogeneous field of equal space-average luminance. Those results, reported in several colloquia, have not as yet been published. I am grateful to Professor Cornsweet for the information he has provided me regarding his unpublished work relating to Talbot's law in the spatial domain, and I am also grateful to an anonymous reviewer for calling that work to my attention.

(Received for publication May 25, 1977; revision accepted February 17, 1978.) 